\title{
Negative Beziehungsaspekte und gesundheitliche Ungleichheiten
}

\author{
Philip Adebahr
}

\section{Überblick}

- Negative Beziehungsaspekte werden definiert als interpersonelle Verbindungen zwischen zwei Personen, die von Ego als belastend wahrgenommen werden. In der Forschung müssen handlungsorientierte, einstellungsorientierte und strukturorientierte Ansätze differenziert werden.

- Stress und Gewalt (körperlich), geringerer Selbstwert und Depression (psychisch) und verändertes Gesundheitsverhalten sind potenzielle gesundheitliche Folgen negativer Beziehungsaspekte. Ob negative Beziehungsaspekte zudem auch gesundheitsförderlich Effekte erzielen (salutogene Perspektive) und inwiefern die Gesundheit umgekehrt auch auf die Entstehung negativer Beziehungsaspekte Einfluss nimmt, bleibt Gegenstand zukünftiger Forschung.

- Es bestehen mehrere theoretische Konzeptionen, um den Zusammenhang zwischen sozioökonomischem Status (SES) und negativen Beziehungsaspekten zu erklären. In diesem Beitrag werden der Diskriminierungs-, der Ressourcen- und der Sozialisationsansatz aufgegriffen und mit empirischen Befunden unterlegt.

- Um den Zusammenhang aller drei Aspkete (zwischen SES, Gesundheit und negativen Aspekten sozialer Beziehungen) hinlänglich zu klären, bedarf es weiterer Forschung auch in Richtung einer salutogenen Perspektive und der Wirkrichtungen der Zusammenhänge.

P. Adebahr $(\bowtie)$

Chemnitz, Deutschland

E-Mail: philip.adebahr@soziologie.tu-chemnitz.de

A. Klärner et al. (Hrsg.), Soziale Netzwerke und gesundheitliche Ungleichheiten, https://doi.org/10.1007/978-3-658-21659-7_5 


\section{$1 \quad$ Negative Beziehungsaspekte - eine Einführung}

Während in der Netzwerkforschung bereits Konzepte für positive Beziehungen breit diskutiert sind, zu nennen wäre hier beispielsweise das Konzept des Sozialkapitals (siehe Kap. „Netzwerktheorie(n)“), gibt es in der Netzwerkforschung bis dato noch kein einheitliches Verständnis von negativen Beziehungen (negative ties). Diese Vielfalt spiegelt sich auch in den diversen Operationalisierungen wider. Labianca (2014, S. 241) fasst zusammen, dass negative Aspekte beispielsweise mit Begriffen wie ,distant relationships, difficult relationships, prefer to avoid, dislike, distrust, conflict, relationship conflict, task conflict, disrupts, excludes socially, being a political adversary, troubled relationships“ erhoben werden. Je nach Theorieposition (z. B. Konflikttheorien, Balancetheorie, Sozialkapitalansatz - näheres im folgenden Absatz) herrscht ein anderes Verständnis von negativen Beziehungsaspekten vor, wobei ein gewisser Konsens in folgenden drei Definitionskriterien vorzufinden ist (vgl. z. B. Borgatti et al. 2014, S. 9; Chauvac et al. 2014, S. 7; Heider 1946, S. 107; Labianca und Brass 2006, S. 597; Laireiter und Lettner 1993). Erstens existiert ein „tie“1 also eine Verbindung zwischen den Akteuren, zweitens weist die Verbindung interpersonelle Spannungen bzw. negative Aspekte (z. B. durch Konkurrenz um Ressourcen, verletzte Reziprozitätserwartungen, u. ä.) auf, welche von Ego als belastend wahrgenommen werden und mit Gefühlen der Abneigung z. B. Angst, Hass, Groll o. ä. verbunden sind. Drittens wird eine dyadische Perspektive eingenommen (Ego-Alter-Relation), welche die Analyse auf multipersoneller Ebene erlaubt, ohne die individuelle Perspektive zu vernachlässigen. Somit kann zwischen gegenseitigen und einseitigen Wahrnehmungen der Beziehung unterschieden und die Entstehung von Emergenz nachvollzogen werden.

Während die Frage der Verbindung (tie) und die dyadische Perspektive noch relativ leicht $\mathrm{zu}$ beantworten sind, existieren hingegen noch Differenzen in der Gegenstandsbestimmung: Was sind negative Verbindungen? Hier stehen sich drei Konzeptionen gegenüber, deren Unterschied primär im Aggregationsgrad der „Verbindung“ deutlich wird: Erstens, die handlungsorientierte Konzeption, in der

\footnotetext{
${ }^{1}$ Der Begriff Verbindung (engl. tie) wird hier vom Begriff Beziehung (engl. relationship) abgegrenzt. Nach Perry et al. (2018, S. 161) kann die Beziehung von Ego zu Alter in eine Zusammenstellung analytisch unterscheidbarer Verbindungen (ties) unterteilt werden. Beziehungen sind folglich das Aggregat der verbindenden und spannungsgeladenen Elemente (ties). In Anlehnung daran, wird im vorliegenden Beitrag von negativen Beziehungsaspekten gesprochen und nicht von negativen Beziehungen.
} 
die einzelnen konflikthaften Interaktionen und Interaktionsketten die Verbindung schaffen. Simmel ist mit seinen Ausführungen über den Streit einer der frühen Vertreter dieser Forschungsperspektive (vgl. Simmel 1908, S. 186-225). Mittlerweile ist der Terminus ,sozialer Konflikt“ weit verbreitet. Obgleich der Konfliktbegriff Anschlussfähigkeit zu vielen wissenschaftlichen Diskursen schafft (z. B. zur Aggressions- und Gewaltforschung), ist er aus analytischer Sicht problembehaftet. Einerseits, da in der soziologischen Konfliktforschung begrifflich weniger zwischen Auseinandersetzungen auf Mikro- (zwischen Einzelpersonen) und Makroebene (zwischen sozialen Gruppen) sowie zwischen den Ebenen (zwischen Einzelpersonen und Gruppen) unterschieden wird. Dies verdeutlicht folgende Definition. Soziale „Konflikte lassen sich [...] als soziale Tatbestände [definieren], an denen mindestens zwei Parteien (Einzelpersonen, Gruppen, Staaten etc.) beteiligt sind, die auf Unterschieden in der sozialen Lage und/oder auf Unterschieden in der Interessenkonstellation der Konfliktparteien beruhen" (Bonacker und Imbusch 2010, S. 69). Es gilt also weitaus trennschärfer zu differenzieren zwischen interpersonellem Konflikt als „Aufeinandertreffen von zwei miteinander unvereinbaren Handlungstendenzen [zwischen Personen]" und innerhalb einer Person (intrapersoneller Konflikt) sowie Intergruppenkonflikte (zwischen sozialen Gruppen) und Intragruppenkonflikte (innerhalb sozialer Gruppen) zu unterscheiden (vgl. Nolting 1998, S. 552). Andererseits ist der Terminus „,sozialer Konflikt“ im Kontext negativer Beziehungsaspekte problematisch, da negative Aspekte sozialer Beziehungen nicht zwangsläufig in Konflikten münden. Konflikthaftes Verhalten ist, neben Loyalität, Vermeidung und Beziehungsabbruch, eine von mehreren Coping-Strategien (dt. Bewältigungsstrategien) für den Umgang mit interpersonellen Spannungen (siehe voice, loyality, neglect, exit bei Rusbult und Zembrodt 1983). Nach diesem Verständnis gehen mit interpersonellen Konflikten logischerweise interpersonelle Spannungen einher, mit interpersonellen Spannungen aber nicht zwangsläufig interpersonelle Konflikte (gleiches gilt für Gewaltphänomene).

\section{Exkurs: Negative Beziehungsaspekte und Gewalt}

Streitbezogene Gewalt ist eine Spezialform konflikthafter Spannungsbewältigung. Der Zusammenhang zwischen negativen Aspekt sozialer Beziehungen und personenbezogener Gewalt ist vermittelt über Aggressivität, welche jedoch nicht zwangsläufig in aggressives, antisoziales Verhalten umgesetzt wird. Zudem gehen nicht jeder Gewalttat negative Beziehungsaspekte zum Opfer voraus (z. B. bei räuberischer Gewalt). Die Bedingungen zur Entstehung von Aggressionen und Gewalttaten weisen 
Schnittstellen mit negativen Aspekten sozialer Beziehungen auf. Beispielsweise ist im General Aggression Modell die Dimension externe „Person“ als Bedingungsfaktor aufgeführt (Allen et al. 2018). Einen Überblick zur Aggressions- und Gewaltforschung bieten Heitmeyer und Hagan (2002) und Bogerts und Möller-Leimkühler (2013). Bei häuslicher Gewalt besteht hingegen eine Verbindung zwischen den Personen (gemeinsames Wohnen), und auch wenn nicht immer eine Absicht unterstellt wird, wird die Tat dennoch als schädigend oder belastend erlebt. Damit erfüllt sie für das Opfer in der Regel die Definitionskriterien negativer Beziehungsaspekte.

Aggregierte Einstellungen bilden die zweite Konzeption von negativen Verbindungen. Danach sind weniger die Einzelsituationen von Bedeutung für die Beschreibung einer „Verbindung“, sondern vielmehr die Gesamtbewertung der Beziehung bzw. Egos Einstellung zu Alter. Einer Definition von Labianca und Brass zufolge werden negative Verbindungen dann als ein andauerndes, wiederkehrendes Set von negativen Bewertungen, Gefühlen und Verhaltensintentionen gegenüber Alter definiert (Labianca und Brass 2006, S. 597). ${ }^{2}$ Labianca und Brass argumentieren, dass Ego ähnlich einem Buchhaltungskonto eine Gesamteinschätzung (mögen/like oder ablehnen/dislike) entwickelt (ein sogenanntes „,negative person schema“; ebd.). Die Gesamtbewertung als ,negative Beziehung" ist bei näherer Betrachtung ebenfalls problembehaftet. Negative Aspekte sind in der Regel mit Ambivalenz verbunden bzw. mit einer Gleichzeitigkeit von positiven und negativen Beziehungsaspekten, da eine rein negative Beziehung nach Klein Ikkink und van Tilburg (1999) aufgrund fehlenden Nutzens in der Regel aufgelöst wird (siehe zu Ambivalenz auch Ajzen 2001; Coser 2009; Lüscher 2011; Simmel 1908, S. 186-225). Entsprechend der Gleichrangigkeit beider Beziehungsaspekte wird es den Individuen schwerfallen, eine ,positive oder negative" Gesamteinschätzung zu geben. $\mathrm{Zu}$ vermuten ist die Verzerrung zugunsten positiver Gesamtbewertungen, da Ego die Beziehung strategisch nutzt und so die positiven Aspekte in den Vordergrund stellt.

\footnotetext{
${ }^{2}$ Damit schließen Labianca und Brass an Heider und die Einstellungsforschung (engl. attitudes) an, die ebenfalls die kognitive, affektive und behaviorale Dimension aufgreifen vgl. Ajzen (2001); Banaji und Heiphetz (2010); Heider (1946), sowie an die, social ledger theorie‘ Labianca und Brass (2006).
} 
Die dritte Konzeption von negativen Verbindungen ist strukturorientiert. Demnach sind negativen Beziehungen jene, die den Zugang zu Ressourcen verhindern (Chauvac et al. 2014, S. 7) oder soziales Kapital reduzieren (Borgatti et al. 2014, S. 9). Die negative Verbindung besteht darin, dass Alter systematisch Verbindungsversuche von Ego blockiert oder zu unterbrechen versucht (zu sich und zu dritten). Die Verhinderung von Zugängen zu Ressourcen impliziert einen Zugangsversuch und somit eine interaktionistische Verbindung. Zusätzlich wird der Blick auf die Beziehungsdyade um die Beziehungstriade erweitert (siehe hierzu Heider 1946 und Kap. „Netzwerktheorie(n)“).

Obgleich der Verweis auf Stigmatisierung, Diskriminierung und Exklusion naheliegt und in der Forschung zu Mobbing (engl. Bullying) ebenfalls von ,negative ties" gesprochen wird (z. B. Kaur und Singh 2015, S. 24), ist die Verbindung zu dieser makrosoziologischen Perspektive nicht klar. Dies liegt daran, dass die Eindeutigkeit der mikrosoziologischen Definitionskriterien (Verbindung, Negativität, Dyaden-Perspektive) in der Abstraktion makrosoziologischer Betrachtung verloren geht. Zur Erläuterung: Das soziologische Begriffsinstrumentarium von Exklusion und Ausgrenzung ist nicht mit negativen Beziehungsaspekten gleichzusetzen. Einerseits kann Diskriminierung vom Forschenden beobachtet werden, aber zugleich den Individuen (Diskriminierender oder Diskriminierter) unbewusst bleiben (keine wahrgenommene Negativität). Andererseits verweist die Einführung der Beziehungstriade darauf, dass Diskriminierung stattfinden kann, obwohl Ego und Alter nicht in direkter Interaktion miteinander stehen. Der Austausch über die diskriminierte Personengruppe führt dazu, dass eine Interaktion mit dieser gar nicht erst in Erwägung gezogen wird (keine Verbindung).

Dass eine Verbindung zwischen Ego und Alter besteht, ist, wie oben dargelegt, ein zentrales Definitionskriterium. Es bedarf jedoch einer Erklärung, warum negative Verbindungen über längere Zeit fortbestehen können. Offer und Fischer (2018, S. 3-6) geben hier zwei Möglichkeiten vor. Erstens werden Beziehungen aus strategischen Kosten-Nutzen-Überlegungen aufrechterhalten. Beispielsweise kann die Beziehung zu einem Augenarzt von strategischem Nutzen sein, wenn Ego häufig Augenbeschwerden hat, die nächste Augenarztpraxis jedoch $50 \mathrm{~km}$ entfernt ist. Hier spart ein kurzes informelles Telefonat möglicherweise den aufwendigen Gang zum Augenarzt. Zweitens verfügt das Individuum nur über einen eingeschränkten Zugang zu einem Pool an Interaktionspartnern und ist daher nicht in der Lage, die belastete Interaktion zu beenden. Letztlich kann eine Beziehung so sehr von Nutzen sein, dass die Kosten, d. h. die negativen Aspekte der Beziehung, billigend in Kauf genommen werden. Beziehungen 
bestehen auch aufgrund schwierig begleichbarer Kosten, sogenannter externer und interner Barrieren, weiter (Hess 2000). Externe Barrieren entstehen außerhalb des Individuums und umfassen dabei die Bindung von Ego und Alter durch institutionelle Zugehörigkeit zu sozialen Gruppen (z. B. Kirche, Vereine, Verwandtschaft), finanzielle Verpflichtungen und Verträge (z. B. gemeinsame Kredite oder Verantwortung für Kinder und Wohnung) oder physische Nähe (z. B. durch Nachbarschaft und Arbeitsplatz) (Bushman und Holt-Lunstad 2009, S. 754; Hess 2003). Interne Barrieren hingegen bestehen nach Hess aus inneren Wünschen und Überzeugungen des Individuums, die zur Aufrechterhaltung einer Beziehung führen, auch wenn diese negative Aspekte beinhalten (Hess 2003). Zur Aufrechterhaltung interner Barrieren tragen demnach bei: Sogenannte self-identity goals, Ziele, welche in der Identität und dem Selbstbild des Individuums verankert sind. Zweitens, die damit teilweise einhergehenden Verantwortungs-, Pflicht- und Verbundenheitsgefühle (sense of commitment), und drittens auch Glaubenssätze und Überzeugungen z. B. von Nächstenliebe und Vergebung (religious beliefs). Hess fügt ergänzend subtile Ängste vor den Folgen der Beziehungsauflösung hinzu (z. B. die Angst, jemanden zu verletzen) (Hess 2000, 2003, 2016).

Zusammenfassend kann festgehalten werden, dass sich negative ties durch eine Verbindung (1) zwischen Ego und Alter (Dyadenperspektive (2)) auszeichnet, die von Ego als belastend wahrgenommen wird (3). Sie sind häufig mit schwer lösbaren Exit-Barrieren verbunden. Zudem bestehen Unterschiede darin, ob die negativen Aspekte als spannungsgeladene Einzelsituationen (interpersonelle Spannungen), als aggregierte Gesamteinschätzung (Einstellung), oder strukturell, als Zugangsbarrieren zu bestimmten Personen und Netzwerken, verstanden werden. Um den aktuellen Forschungsdiskurs in all seinen Facetten aufgreifen zu können, wird von negativen Aspekten im Allgemeinen gesprochen und sowohl Einzelsituationen als auch Gesamteinschätzungen betrachtet. Die zentrale Frage dieses Kapitels (Negative Beziehungsaspekte und gesundheitliche Ungleichheiten) besteht darin, den Forschungsstand über den Beitrag negativer Beziehung zur Reproduktion gesundheitlicher Ungleichheit zu eruieren. Nachdem in diesem Abschnitt dargelegt wurde, was unter negativen Beziehungen verstanden wird, erfolgt in Abschn. 2 die Darlegung des Zusammenhangs zwischen negativen Beziehungen und verschiedenen Gesundheitsparametern. Auf die Frage, inwiefern dieser Einfluss sozial ungleich verteilt ist, wird in Abschn. 3 eingegangen. Der Beitrag schließt in Abschn. 4 mit einer Zusammenfassung und einem Ausblick, in dem die wesentlichen Desiderata zur Forschung über negative Beziehungsaspekte und gesundheitlicher Ungleichheit angesprochen werden. 


\section{Negative Beziehungsaspekte und Gesundheit}

Obgleich negative Beziehungsaspekte seltener sind als positive (Labianca und Brass 2006; Offer und Fischer 2018), deuten empirische Befunde darauf hin, dass in bestimmten Fällen Belastungen aus sozialen Beziehungen einen größeren gesundheitsbezogenen Effekt erzeugen als positive Beziehungsaspekte (Rook 1998; Brooks und Dunkel Schetter 2011). Die Frage, wie häufig negative Verbindungen auftreten, wird unterschiedlich beantwortet. Je nach Sample und Operationalisierung, ergibt sich eine Prävalenz ,negativer Beziehung“ in persönlichen Netzwerken von bis zu 8 Prozent (Zusammengetragen von Labianca und Brass 2006, S. 597). Neuere Erkenntnisse weisen darauf hin, dass der Anteil in der allgemeinen Bevölkerung weitaus größer sein könnte. Offer und Fischer (2018, S. 1) berichten für die erste Welle der Studie UCNets (University of California Social Networks Study), dass $15 \%$ aller Beziehungen als ,,sometimes demanding or difficult" eingeschätzt werden. Untersucht wurden zwei Kohorten, 21- bis 30-Jährige und 50- bis 70-Jährige. Bezogen auf die Effekte weist Rook (1984) für ältere verwitwete Frauen nach, dass die Anzahl an belastenden Beziehungen das psychische Wohlbefinden stärker beeinflussen als die Anzahl unterstützender Beziehungen. Ähnliches bezeichnen Cacioppo und KollegInnen mit dem Begriff ,negativity bias", nachdem negativen Informationen im Gehirn größere Bedeutung zugeschrieben wird als positiven (Cacioppo und Gardner 1999; Ito et al. 1998).

\subsection{Negative Aspekte sozialer Beziehungen und körperliche Gesundheit}

Insofern negative Aspekte sozialer Beziehungen aufgrund starker Exit-Barrieren oft dauerhaft sind, kann hier von einem chronischen sozialen Stressor gesprochen werden. Dies führt zu wiederkehrender oder langanhaltender Aktivierung des Körpers durch die Stressreaktion. Das meint, der Körper wird in den ,fight-orflight"-Zustand versetzt (Cannon 1932). ${ }^{3}$ Es erfolgt eine Steigerung des Herzschlags und Blutdrucks, verstärkte Lungenventilation sowie Freisetzung von

\footnotetext{
${ }^{3}$ Mittlerweile ist bekannt, dass die Stressreaktion vom Kontext, seiner Wahrnehmung sowie von individuellen und sozialen Faktoren abhängt. Beispielsweise wird die weibliche Stressreaktion als ,tend-and-befriend'-Reaktion (deutsch: sich kümmern und Anfreunden) beschrieben (zuerst Taylor et al. 2000; vertiefend siehe Seidel et al. 2013).
} 
Fettsäuren und Glukose (Dawans und Heinrichs 2018). Zudem werden die Verdauung und Antikörperproduktion gehemmt (ebd.). Das dauerhafte Bestehen stressbedingter Prozesse im Körper wird als ,allostatic load“ bezeichnet. Dauerhaft als negativ empfundene Interaktionen stehen in Verbindung mit allostatischen Belastungen (Seeman et al. 2014). Die stressbedingte Krankheitsentstehung (,Allostase“) wurde bereits breit beforscht (z. B. Rensing 2013). Bekannt ist der Zusammenhang zwischen sozialem Stress und Herzkreislauferkrankungen z. B. Bluthochdruck (Sneed und Cohen 2014), koronaren Herzerkrankungen (Orth-Gomér 2007, 2009; Vogli et al. 2007) oder Schlaganfällen (Tanne et al. 2004). Auch das Endokrinsystem (Hormonhaushalt) wir durch negative Interaktionen verändert. Andauernde negative Interaktionen stehen z. B. in Verbindung mit hohem Cortisolspiegel, welcher das Immunsystem schwächt und die Anfälligkeit für Krankheiten erhöht (Dawans und Heinrichs 2018; Siegrist 2018). Auch die Wundheilung (Cytokine-Produktion, IL-6, TNF $\alpha$, IL-1 $\beta$ ) bzw. die damit verbundene Nachproduktion und Erneuerung von absterbenden Zellen wird von sozialem Stress gehemmt (Kiecolt-Glaser et al. 2005; Wright und Loving 2011). Als weitere körperliche Folge negativer Aspekte sozialer Beziehungen können körperliche Verletzungen aufgrund von physischer Gewalt angeführt werden. Wie in Abschn. 1 dargelegt, kann Gewalt eine Copingstrategie (Extremform konflikthaften Copings) für interpersonelle Spannungen sein. Laut einer Studie von Eggert et al. (2018, S. 8) erfahren beispielsweise elf Prozent der pflegenden Angehörigen körperliche Gewalt durch die Gepflegten in den letzten sechs Monaten. Wobei zwölf Prozent angaben, körperliche Gewalt gegen die pflegebedürftige Person ausgeübt zu haben (ebd.). Dabei wurde nur Gewalt erfasst, die beabsichtigt, also personengebunden ist sowie als schädigend erlebt wird und somit den Kriterien negativer Beziehungsaspekte entspricht (vgl. Eggert et al. 2018, S. 6).

\subsection{Negative Aspekte sozialer Beziehungen und mentale Gesundheit}

Psychische Gewalt ist im Vergleich zur körperlichen Gewalt in der häuslichen Pflege häufiger anzutreffen. $45 \%$ der pflegenden Angehörigen berichten laut Eggert et al. von psychischer Gewalt durch die Gepflegten in den letzten sechs Monaten (2018, S. 8). Wobei $32 \%$ angeben, selber psychische Gewalt gegen die pflegebedürftige Person ausgeübt zu haben (ebd.). An dieser Stelle sei auch auf die Gewalt zwischen Eltern und Kindern, zwischen Geschwistern sowie in der 
Partnerschaft als weitere Formen häuslicher Gewalt verwiesen (Lamnek et al. 2013). 2017 wurden laut Statistik des Bundeskriminalamtes 138.893 Personen Opfer partnerschaftlicher Gewalt (Bundeskriminalamt 2018, S. 28). Sowohl körperliche als auch psychische Gewalt haben zum Teil in Form von Psychotraumata Auswirkungen auf die mentale Gesundheit und spielen dann eine zentrale Rolle bei der Entstehung psychischer Störungen (Pape und Binder 2014).

Aber auch geringere soziale Belastung, wie kleine Streitereien und Auseinandersetzungen, können mit schlechter psychischer Gesundheit einhergehen. Einen Erklärungsansatz bietet die Soziometer-Theorie, nach welcher Selbstwert ein Indikator (Soziometer) für die eigene soziale Integration darstellt (Leary 2005). Der Soziometer-Theorie folgend, können interpersonelle Konflikte und Zurückweisungen die psychologischen Ressourcen (z. B. Selbstbewusstsein und Selbstwirksamkeitseinschätzung) herabsetzen (vgl. Leary 2005, 2012). Andererseits ist ebenfalls belegt, dass niedriger Selbstwert die Wahrscheinlichkeit interpersoneller Konflikte erhöht (Kiviruusu et al. 2016, S. 2). Zur kritischen Empirie-fokussierten Auseinandersetzung mit dieser Theorie siehe die Metaanalyse von Blackhart et al. (2009). Beide Ressourcen (Selbstbewusstsein und Selbstwirksamkeitseinschätzung) sind negativ mit Depression assoziiert. Stafford et al. (2011) finden auf Basis einer Panelstudie etwa Zusammenhänge zwischen negativen sozialen Interaktionen in engen Beziehungen (Familie, enger Freundeskreis) und Depressionen. Positiver Austausch ist hingegen nicht zwingend mit weniger Depression assoziiert (ähnlich siehe Lincoln et al. 2010).

\subsection{Negative Aspekte sozialer Beziehungen und Gesundheitsverhalten}

Ungünstige Gesundheitseffekte gehen nicht ausschließlich von der Stresssituation, d. h. den negativen Aspekten sozialer Beziehungen an sich, aus, sondern auch von Egos Reaktion (Verhalten) auf den negativen Beziehungsaspekt. Aus soziologischer Perspektive wirken die Individuen des sozialen Netzwerks als Kontrollinstanz, und negative Beziehungsaspekte bilden eine Form der Sanktionierung, die Personen $\mathrm{zu}$ einem bestimmten Verhalten veranlassen sollen (vgl. Peuckert 2006). Wie Personen mit Sanktionierung umgehen, hängt von der gewählten Bewältigungsstrategie (Copingstrategie) ab. Eine Systematisierung von Bewältigungsstrategien bietet beispielsweise das Dualaxiale Copingmodell 
(Hobfoll 1998). ${ }^{4}$ Darin wird zwischen aktivem/passiven sowie pro-/antisozialem Coping (Bewältigungsverhalten) unterschieden. Eine pauschale Antwort auf die Frage nach dem Zusammenhang der einzelnen Copingstrategien mit Gesundheit stößt, in Hinblick auf die vielen Einzelphänomene, schnell an seine Grenzen. Im Folgenden werden die vier Typen am Beispiel Fat-Shaming erläutert. Fat-Shaming bezeichnet die ,diskreditierende, meist öffentliche Äußerung gegenüber vermeintlich übergewichtigen Personen in Form von konkreter Kritik aufgrund des Gewichts, die als beleidigend gemeint oder verstanden wird" (Adebahr und Lehmann 2017, S. 2).

- Passiv prosoziales Coping besteht in vorsichtigem Handeln, z. B. in der Vermeidung des Kontakts mit dem Beleidigenden.

- Passives antisoziales Coping umfasst instinktives Handeln (passiv), bei der die spontane Reaktion negative Konsequenzen mit sich bringen kann (antisozial). Zum Beispiel sinkt durch das Fat-Shaming die Motivation der übergewichtigen Person, sich sportlich zu betätigen, Vorsorgeuntersuchungen in Anspruch zu nehmen, und es entsteht eine ablehnende Haltung zu Diäten (Alegria Drury und Louis 2002; Puhl und Brownell 2006; Sykes und McPhail 2008).

- Aktives prosoziales Coping besteht in der Suche nach Unterstützung und der Bildung von Koalitionen. Bei Fat-Shaming könnte das dem Anschluss an die Fat-Positive-Bewegung gleichkommen.

- Aktiv antisoziales Coping zeigt sich beispielsweise durch Gewalttaten (aktiv) mit entsprechenden Verletzungsabsichten (antisozial). Beispiele bilden das Beleidigen oder Schlagen des Fat-Shamers.

Copingstrategien können gesundheitsförderliches Verhalten verstärken. Die Teilnahme an einer Selbsthilfegruppe für Übergewichtige erhöht beispielsweise die Wahrscheinlichkeit der Gewichtsreduktion. Genauso könnten aber auch gesundheitshemmende Verhaltensweisen eingeleitet werden. So senkt Fat-Shaming beispielsweise die Wahrscheinlichkeit, sich sportlich zu betätigen (Sykes und McPhail 2008). Welches Verhalten gewählt wird, gilt es für jedes Phänomen mithilfe sozialer Handlungstheorien und empirischer Forschung einzeln zu erklären (beispielhaft siehe Rook et al. 2011).

\footnotetext{
${ }^{4}$ Das Multiaxiale Copingmodell enthält eine zusätzliche Achse (direkt vs. indirekt), welche primär kulturspezifische Unterschiede beim Coping abbildet (Hobfoll 1998). Kulturunterschiede stehen jedoch nicht im Fokus dieses Beitrags, weshalb das Dualaxiale Copingmodell vorgestellt wird.
} 
Negative Aspekte sozialer Beziehungen tragen zusammenfassend folgendermaßen zur Reproduktion gesundheitlicher Ungleichheit bei:

1. Auf körperlicher Ebene durch Stress und Verletzung aufgrund von Gewalt.

2. Auf psychischer Ebene durch verringerte mentale Gesundheit z. B. in Form von geringerem Selbstwert und höherem Depressionsrisiko.

3. In bestimmten Fällen kann der soziale Druck durch negative Beziehungsaspekte Veränderungen des (Gesundheits-)Verhaltens bewirken, wobei die gesundheitshemmenden oder -förderlichen Effekte von der Einzelsituation abhängen.

\section{Sozialer Status, negative Beziehungsaspekte und Gesundheit}

Inwiefern negative Aspekte sozialer Beziehungen, und somit auch die in Abschn. 2 angeführten gesundheitlichen Zusammenhänge, nach Dimensionen sozialer Ungleichheit variieren, ist die leitende Frage dieses Beitrags. Eine Studie, die systematisch den Einfluss negativer Aspekte sozialer Beziehungen auf den Zusammenhang zwischen sozioökonomischem Status (SES) und Gesundheitsparameter analysiert ist nicht bekannt. Aus diesem Grund wird in diesem Abschnitt der These nachgegangen, dass soziale Ungleichheiten mit negativen Aspekten sozialer Beziehungen zusammenhängen. Ziel ist es, durch Abschn. 2 und 3 die These $\mathrm{zu}$ untermauern, dass niedriger SES mit mehr negativen Beziehungsaspekten einhergeht und damit die niedrigere Gesundheit bei geringem SES zum Teil erklärt wird.

Krause et al. (2008, S. 1013) stellen anhand zweier Datensätze mit US-amerikanischen Senioren heraus, dass finanzielle Schwierigkeiten mit mehr negativen Interaktionen einhergehen (handlungsorientierte Konzeption, siehe Abschn. 1). Zudem finden sie über beide Datensätze keine Bildungseffekte im Zusammenhang mit negativen Interaktionen. Des Weiteren deuten ihre Ergebnisse darauf hin, dass die Auswirkungen persönlicher wirtschaftlicher Schwierigkeiten bei mehr negativer sozialer Interaktion stärker gesundheitshemmend wirken. Negative Interaktionen in Form von „,keine Hilfe bekommen, wenn sie erwartet wird“ verstärken zusätzlich den Effekt von finanziellen Spannungen auf die subjektive Gesundheit (ebd.).

Offer und Fischer (2018) fragen hingegen nach Personen, die als besonders schwierig wahrgenommen werden (gesamteinschätzungsorientierte Konzeption siehe Abschn. 1). Danach zeigen die Ergebnisse der California Social Network Study (UCNets) bei anderer Operationalisierung gegenläufige Effekte. Gegenüber dem eben berichteten Effekt finanzieller Spannungen, wird in der multivariaten 
Analyse von Offer und Fischer der Zusammenhang zwischen Einkommen und der Anzahl von als schwierig eingeschätzten Personen nicht signifikant, wohingegen Bildung einen signifikanten Zusammenhang mit als schwierig eingeschätzten Personen aufweist. In der Altersgruppe zwischen 50 und 70 Jahren haben Personen mit einem Bildungsabschluss unter Bachelor weniger Personen im Netzwerk, die sie als schwierig einschätzen, allerdings gleichzeitig mehr Personen, die sie als ambivalent wahrnehmen (Offer und Fischer 2018). ${ }^{5}$ Zusammenfassend korrelieren existenzielle finanzielle Schwierigkeiten unabhängig von der Bildung mit negativen Interaktionen, wohingegen die Anzahl als schwierig wahrgenommener Personen vom Bildungsgrad (unabhängig vom Einkommen) abhängt. Nach Vogli et al. (2007) sind zudem Personen in statusniedrigeren Beschäftigungsverhältnissen eher negativen Aspekten sozialer Beziehungen ausgesetzt als Personen in höheren Statusgruppen. Nachdem nun einige empirsche Befunde dargelegt sind, werden im Folgenden drei mögliche Erklärungsanätze umrissen.

Eine Argumentation, die den Zusammenhang zwischen finanziellen Problemen und negativen Aspekten sozialer Beziehungen zu erklären versucht, stammt von Krause et al. (2008). Sie argumentieren, dass die eingeschränkte Ressourcenausstattung (Erklärungsansatz 1) und Hilfsbedürftigkeit von Personen mit niedrigem sozioökonomischem Status (SES) die Erbringung einer adäquaten Gegenleistung erschweren, was letztlich eher zu Belastungen und sozialen Spannungen führt. Eng umgrenzte soziale Netzwerke tendieren zur Statushomogenität der Mitglieder (Belle 1983; Phan et al. 2009). ${ }^{6}$ Das bedeutet, dass im sozialen Netzwerk statusniedriger Personen eher Personen mit finanziellen Schwierigkeiten vorhanden sind, was wiederum die Wahrscheinlichkeit für das Auftreten sozialer Spannungen durch finanzielle Schwierigkeiten erhöht. Hobfoll (1998, S. 208) bezeichnet diesen tief greifenden Effekt als ,pressure cooker effect“" Wenn Personen selber zu wenig Ressourcen besitzen, um Probleme zu bewältigen, wird es ihnen schwerfallen, andere bei deren Problemen zu unterstützen (Krause et al. 2008). Empirisch untermauert wird dies durch die Beobachtung, dass die Unterstützung aus Netzwerken statusniedriger Personen oft geringer ausfällt, sporadisch, nicht verlässlich und von Spannungen geprägt ist (Offer 2012, S. 789).

Diesem Erklärungsansatz stehen andere entgegen. Im Sinne sozialisationstheoretischer Überlegungen (Erklärungsansatz 2) kann beispielsweise argumentiert werden, dass Personen niedriger sozialer Statusgruppen eher Konflikte

\footnotetext{
${ }^{5}$ Ergebnisse für die Altersgruppe 21-30 sind nicht signifikant bezogen auf die Bildung. ${ }^{6}$ Der Prozess, nachdem sich Personen nach gleichen Merkmalen in soziale Gruppen finden, wird in der Netzwerkforschung als Homophilie bezeichnet Lin (2000).
} 
vorgelebt bekommen. Sie versuchen deshalb auch häufiger, soziale Spannung durch konflikthaftes Verhalten zu lösen (weniger durch kooperatives Verhalten). Inwiefern drohender Ressourcenverlust in unteren sozialen Schichten als existenzbedrohender angesehen wird (als in höheren Schichten) und deshalb die Reaktion radikaler (anti- und prosozial) ausfällt, bleibt zu prüfen. Richtungsweisende Ergebnisse sind bei Greitemeyer und Sagioglou (2018) dargelegt. Sie zeigen, dass in vier von fünf betrachteten Studien niedriger SES mit aggressiverem Verhalten einhergeht. ${ }^{7}$

Aus der Schulforschung ist bekannt, dass Prozesse sozialer Schließung und Diskriminierung in Form von Mobbing sozial ungleich verteilt sind. Dies ist ein dritter Erklärungsansatz für den Zusammenhang von SES und negativen Aspekten sozialer Beziehungen. Demnach werden Personen mit niedrigem SES eher diskriminiert. Auch wenn SES ein schwacher Prädiktor für Mobbing in der Schule ist, zeigt die Metastudie von Tippett und Wolke (2014) einen signifikant positiven Zusammenhang zwischen niedrigem SES und der Wahrscheinlichkeit, Opfer von Mobbing zu sein auf. Ähnliches gilt für die Arbeitswelt. Tsuno et al. (2015) berichten einen signifikanten Zusammenhang zwischen hohem subjektivem sozioökonomischen Status und geringem Mobbing. Dieser Ansatz ist aus Netzwerkforschungsperspektive besonders interessant, da die Spannungen primär von den Alteri und nicht von den Egos ausgehen, was die Dyaden und sozialen Netzwerke stärker in den Blick nimmt.

Das ABC-X-Modell integriert alle genannten Perspektiven und fasst diesen Abschnitt zusammen (McCubbin und Patterson 1983). Die Belastung durch negative Beziehungsaspekte $(\mathrm{X})$ hängt demnach von der Art der Stressoren (A) (z. B. Diskriminierung, Ansatz 3), den bestehenden Ressourcen (B) (Ansatz 1) und der Interpretation des Stressors (C) (geprägt durch Sozialisation, Ansatz 2) ab (McCubbin und Patterson 1983).

\section{$4 \quad$ Fazit und Diskussion}

Ziel des Beitrags war es, den Forschungsstand über den Beitrag negativer Beziehung zur Reproduktion gesundheitlicher Ungleichheit zu eruieren. Hierzu wurden die Begriffe rund um „negative ties“ (negative Beziehungsaspekte)

\footnotetext{
${ }^{7}$ Zusätzlich deuten Studien an (z. B. Piff et al. 2010), dass niedriger SES ebenfalls mit stärkerem prosozialem Verhalten einhergeht. Diese Feststellung erweist sich mit Blick auf Greitemeyer und Sagioglou (2018) allerdings als eingeschränkt reliabel.
} 
erörtert, soziologische und psychologische Konzepte eingeführt sowie mögliche Zusammenhänge mit Gesundheit und sozialem Status aufgezeigt und anhand bestehender Theorien diskutiert. Es wurde herausgearbeitet, dass sich negative Beziehungsaspekte durch eine Verbindung (tie) von Ego und Alter (Dyade) auszeichnet, die interpersonelle Spannungen aufweist (Negativität). Sie gehen häufig mit nicht lösbaren Exit-Barrieren einher. Negative Aspekte sozialer Beziehungen wirken sich über Gewalttaten und Stress auf den Körper aus, mit entsprechenden Konsequenzen für die körperliche und mentale Gesundheit (niedrigerer Selbstwert und höheres Depressionsrisiko). Zudem beeinflussen sie, je nach gewählter Copingstrategie, das Gesundheitsverhalten (gesundheitshemmend oder förderlich). Einen integrierenden Erklärungsansatz für den Zusammenhang zwischen SES und negativen Beziehungsaspekten bietet das ABC-X-Modell (McCubbin und Patterson 1983). Auch wenn das Einkommen empirisch keinen signifikanten Zusammenhang mit als schwierig eingestuften Personen zeigt, sind finanzielle Spannungen mit mehr negativen Interaktionen assoziiert. Zudem geht niedriger SES (schwacher Prädiktor) mit einer erhöhten Wahrscheinlichkeit, Opfer von Mobbing zu werden und mehr aggressives Verhalten zu zeigen, einher. Eine Analyse des konkreten Zusammenhangs, also inwiefern negative Beziehungsaspekte die Korrelation zwischen SES und Gesundheit erklären, ist nicht bekannt. Die Ausführung in Abschn. 2 und 3 untermauern die These, dass der Einfluss von sozialem Status auf die Gesundheit zum Teil durch den Anteil negativer Beziehungen erklärt werden kann. Auf Basis der bisherigen Erkenntnisse wird dieser Erklärungsanteil allerdings vergleichsweise gering eingeschätzt.

In den bisherigen Ausführungen sind schon erhebliche Forschungslücken deutlich geworden, an dieser Stelle soll auf einige weitere interessante Aspekte und Limitationen eingegangen werden. In der Forschung zu negativen Aspekten sozialer Beziehungen gibt es eine Vielzahl verschiedener Begriffe und Messinstrumente, die alle ähnliche Aspekte bezeichnen, jedoch noch nicht systematisch zusammengeführt wurden. Wenn negative Aspekte sozialer Beziehungen als Sammelbegriff für viele unterschiedliche Phänomene verstanden werden, lohnt es sich, diese zu differenzieren und zu schauen, welche negativen Aspekte es gibt (z. B. Vermeidung, Konflikt, Gewalt) und wie sie im Einzelnen mit Gesundheit und sozialer Ungleichheit assoziiert sind. Besonders auf das Coping durch Vermeidung und dessen Gesundheitsfolgen wurde in diesem Beitrag kaum eingegangen.

An den dargelegten Ausführungen zum Zusammenhang zwischen negativen Beziehungsaspekten und Gesundheit können ausblickend vier Diskussionspunkte angemerkt werden. Der erste Diskussionspunkt besteht in der postulierten Wirkrichtung des Zusammenhangs. Soziale Spannungen wirken nicht nur auf die Gesundheit, sondern die Gesundheit wirkt auch auf soziale Spannungen. Dies 
wird in der Forschung wenig beachtet. In einer Studie mit Daten des SOEP der Wellen 2002 bis 2008 wurde beispielsweise herausgefunden, dass eine Verschlechterung der Gesundheit eine signifikante Verringerung der Zufriedenheit mit den sozialen Kontakten nach sich zieht (Kriwy und Nisic 2012). Zudem ist bekannt, dass der Gebrauch verschiedener Drogen (Alkohol, Kokain) Aggression fördert (Ntounas et al. 2018). Auch bezüglich des Zusammenhangs von negativen Beziehungsaspekten mit sozialer Ungleichheit konnte die Fokussierung der Argumentation auf eine Wirkrichtung festgestellt werden (soziale Ungleichheiten wirken demnach auf negative Beziehungsaspekte). Um zu ermitteln, inwiefern negative Aspekte sozialer Beziehungen zu sozialer Ungleichheit beitragen vice versa, sind zeitsensible Daten oder Experimentaldesigns notwendig.

Zweitens arbeiten, besonders was die Gesundheitseffekte betrifft, nur sehr wenige Studien mit dem methodischen Instrumentarium der Netzwerkanalyse, was weitere Erkenntnisse ermöglicht (zweiter Diskussionspunkt). Aus Netzwerkperspektive interessant ist die Frage, inwiefern negative Beziehungsaspekte zur Bildung von Gruppen und Netzwerkgrenzen beitragen, inwiefern sich Stress und negative Beziehungsaspekte in sozialen Netzwerken verbreiten (soziale Ansteckung) und inwiefern dadurch gestresste Gesamtnetzwerke oder negative-tie-Netzwerke entstehen. Besonders interessant vor der Prozessperspektive ist die These Cosers, dass Konflikte die Kontrahenten miteinander vertrauter machen, gemeinsame Normen entstehen lassen und damit den Aufbau von Netzwerken zwischen ihnen langfristig fördern können (Coser 2009, S. 144-154). Darüber hinaus sieht er Konflikte selbst als soziale Beziehung, insofern dadurch immer wieder Machtverhältnisse ausgehandelt werden (Coser 2009, S. 160-165).

Des Weiteren sieht Rook (2015) zukünftigen Forschungsbedarf bezüglich des Zusammenwirkens von positivem und negativem Austausch (dritter Diskussionspunkt). Zum Beispiel bezüglich der Frage, wie viele und welche positiven Aspekte einen negativen Beziehungsaspekt aufwiegen können.

In der Forschung zu sozialen Konflikten wird häufig deren Nutzen hervorgehoben (Bark 2012; Coser 2009; Simmel 1908), dies gilt es auch für negativen Beziehungsaspekte zu prüfen und mögliche gesundheitsförderliche Effekte zu eruieren (Diskussionspunkt vier). Inwiefern mit negativen Aspekten - z. B. durch Abgrenzung und Aufbau von Identität (Jetten et al. 2017), Aktivierung, Motivationssteigerung, oder die Ablenkung von chronischen Krankheiten gesundheitsförderliche Effekte bestehen, könnten Fragen einer solchen salutogenen Perspektive sein. Hierbei wird vermutlich dem individuellen Umdeutungsprozess von Disstress zu Eustress eine zentrale Rolle beikommen. Letztendlich gilt es, negative Beziehungsaspekte werturteilsfrei als Teil eines Prozesses interpersoneller Spannung und Entspannung zu verstehen, mit statischen und dynamischen Phasen (ähnlich siehe Bark 2012, S. 11; Rüssmann et al. 2015, S. 501). 


\section{Leseempfehlungen}

Bonacker, T. (Hrsg.) (2008): Sozialwissenschaftliche Konflikttheorien. Eine Einführung. ISBN 9783531161808. In diesem Sammelband werden die zentralen sozialwissenschaftichen Theorien der Soziologie, Psychologie und Politikwissenschaften verständlich aufgearbeitet und mit Anwendungsbeispielen versehen.

Laireiter, A.-R. \& Lettner, K. (1993), Belastende Aspekte sozialer Netzwerke und sozialer Unterstützung. In A.R. Laireiter (Hrsg.), Soziales Netzwerk und soziale Unterstuitzung. Konzepte, Methoden und Befunde. ISBN 3456824025. Hier werden erstmals die Belastenden Aspekte sozialer Unterstützung systematisch aufgearbeitet.

Rook, K. S. (2015), Social Networks in Later Life. Weighing Positive and Negative Effects on Health and Well-Being. Current directions in psychological science, https://doi.org/10.1177/0963721414551364. Dieser Beitrag bietet einen Überblick über die Forschung zu Gesundheitseffekten von positiven und negativen Aspekten sozialer Netzwerke.

\section{Literatur}

Adebahr, P., \& Lehmann, J. (2017). Die soziale Akzeptanz von „Fat Shaming“ als Akt der Stigmatisierung von Übergewichtigen. In S. Lessenich (Hrsg.), Geschlossene Gesellschaften. Verhandlungen des 38. Kongresses der Deutschen Gesellschaft für Soziologie in Bamberg 2016. http://publikationen.soziologie.de/index.php/kongressband_2016/ article/view/599/pdf_140. Zugegriffen: 28. Febr. 2019.

Ajzen, I. (2001). Nature and operation of attitudes. Annual Review of Psychology, 52, 27-58.

Alegria Drury, C. A., \& Louis, M. (2002). Exploring the association between body weight, stigma of obesity, and health care avoidance. Journal of the American Academy of Nurse Practitioners, 14(12), 554-561.

Allen, J. J., Anderson, C. A., \& Bushman, B. J. (2018). The general aggression model. Current Opinion in Psychology, 19, 75-80.

Banaji, M. R., \& Heiphetz, L. (2010). Attitudes. In S. T. Fiske, D. T. Gilbert, G. Lindzey, \& A. E. Jongsma (Hrsg.), Handbook of social psychology (S. 353-393). Hoboken: Wiley.

Bark, S. (2012). Zur Produktivität sozialer Konflikte. Wiesbaden: Springer VS.

Belle, D. E. (1983). The impact of poverty on social networks and supports. Marriage \& Family Review, 5(4), 89-103.

Blackhart, G. C., Nelson, B. C., Knowles, M. L., \& Baumeister, R. F. (2009). Rejection elicits emotional reactions but neither causes immediate distress nor lowers self-esteem. A meta-analytic review of 192 studies on social exclusion. Personality and Social Psychology Review, 13(4), 269-309. 
Bogerts, B., \& Möller-Leimkühler, A. M. (2013). Neurobiologische Ursachen und psychosoziale Bedingungen individueller Gewalt. Der Nervenarzt, 84(11), 1329-1344. https:// link.springer.com/content/pdf/10.1007/s00115-012-3610-x.pdf. Zugegriffen: 10. Jan. 2019.

Bonacker, T., \& Imbusch, P. (2010). Zentrale Begriffe der Friedens- und Konfliktforschung. Konflikt, Gewalt, Krieg, Frieden. In P. Imbusch \& R. Zoll (Hrsg.), Friedens- und Konfliktforschung. Eine Einführung (S. 67-142). Wiesbaden: VS.

Borgatti, S. P., Brass, D. J., \& Halgin, D. S. (2014). Social network research. Confusions, criticisms, and controversies. In D. J. Brass, G. Labianca, A. Mehra, D. S. Halgin, \& S. P. Borgatti (Hrsg.), Research in the sociology of organizations (Bd. 40, S. 1-29). Bradford: Emerald.

Brooks, K. P., \& Dunkel Schetter, C. (2011). Social negativity and health. Conceptual and measurement issues. Social and Personality Psychology Compass, 5(11), 904-918.

Bundeskriminalamt. (2018). Partnerschaftsgewalt. Kriminalstatistische Auswertung. Berichtsjahr 2017, Bundeskriminalamt. https://www.bka.de/SharedDocs/Downloads/ DE/Publikationen/JahresberichteUndLagebilder/Partnerschaftsgewalt/Partnerschaftsgewalt_2017.pdf?_blob=publicationFile\&v=2. Zugegriffen: 28. Febr. 2019.

Bushman, B. B., \& Holt-Lunstad, J. (2009). Understanding social relationship maintenance among friends. Why we don't end those frustrating friendships. Journal of Social and Clinical Psychology, 28(6), 749-778.

Cacioppo, J. T., \& Gardner, W. L. (1999). Emotion. Annual Review of Psychology, 50, 191214.

Cannon, W. B. (1932). The wisdom of the body. New York: Norton.

Chauvac, N., Cloutier, L., Defossez, A., Akermann, G., \& Federico, A. d. (2014). Negative ties, lost ties, latent ties. Bulletin of Sociological Methodology/Bulletin de Méthodologie Sociologique, 121(1), 5-9.

Coser, L. A. (2009). Theorie sozialer Konflikte. Wiesbaden: VS Verlag.

de Vogli, R., Chandola, T., \& Marmot, M. G. (2007). Negative aspects of close relationships and heart disease. Archives of Internal Medicine, 167(18), 1951-1957.

Eggert, S., Schnapp, P., \& Sulmann, D. (2018). Aggression und Gewalt in der informellen Pflege, Zentrum für Qualität in der Pflege. https://www.zqp.de/wp-content/uploads/ ZQP_Analyse_Gewalt_informelle_Pflege.pdf. Zugegriffen: 10. Jan. 2019.

Gleason, M. E. J., \& Iida, M. (2015). Social support. In M. Mikulincer, P. R. Shaver, J. A. Simpson \& J. F. Dovidio (Hrsg.), APA Handbook of Personality and Social Psychology, Volume 3: Interpersonal Relations (S. 351-370). Washington, DC: American Psychological Association.

Greitemeyer, T., \& Sagioglou, C. (2018). Does low (vs. high) subjective socioeconomic status increase both prosociality and aggression? Social Psychology, 49(2), 76-87.

Heider, F. (1946). Attitudes and cognitive organization. The Journal of Psychology, 21, $107-112$.

Heitmeyer, W., \& Hagan, J. (2002). Internationales Handbuch der Gewaltforschung. Wiesbaden: VS Verlag.

Hess, J. A. (2000). Maintaining nonvoluntary relationships with disliked partners. An investigation into the use of distancing behaviors. Human Communication Research, 26(3), 458-488. 
Hess, J. A. (2003). Maintaining undesired relationships. In D. J. Canary \& M. Dainton (Hrsg.), Maintaining realtionships through communication. Relational, contextual, and cultural variations (S. 103-124). Mahwah: Erlbaum.

Hess, J. A. (2016). Distance regulation in personal relationships. The development of a conceptual model and a test of representational validity. Journal of Social and Personal Relationships, 19(5), 663-683.

Hobfoll, S. E. (1998). Stress, culture, and community. The psychology and philosophy of stress (The Plenum series on stress and coping). New York: Plenum Press.

Ito, T. A., Larsen, J. T., Smith, N. K., \& Cacioppo, J. T. (1998). Negative information weighs more heavily on the brain. The negativity bias in evaluative categorizations. Journal of Personality and Social Psychology, 75(4), 887-900.

Jetten, J., Haslam, S. A., Cruwys, T., Greenaway, K. H., Haslam, C., \& Steffens, N. K. (2017). Advancing the social identity approach to health and well-being. Progressing the social cure research agenda. European Journal of Social Psychology, 47(7), 789-802.

Kaur, M., \& Singh, S. (2015). Analyzing negative ties in social networks. A survey. Egyptian Informatics Journal, 17(1), 21-43.

Kiecolt-Glaser, J. K., Loving, T. J., Stowell, J. R., Malarkey, W. B., Lemeshow, S., Dickinson, S. L., \& Glaser, R. (2005). Hostile marital interactions, proinflammatory cytokine production, and wound healing. Archives of General Psychiatry, 62(12), 1377-1384.

Kiviruusu, O., Berg, N., Huurre, T., Aro, H., Marttunen, M., \& Haukkala, A. (2016). Interpersonal conflicts and development of self-esteem from adolescence to mid-adulthood. A 26-year follow-up. PloS one, 11(10), e0164942.

Klein Ikkink, K., \& van Tilburg, T. (1999). Broken ties. Reciprocity and other factors affecting the termination of older adults' relationships. Social Networks, 21(2), 131-146.

Krause, N., Newsom, J. T., \& Rook, K. S. (2008). Financial strain, negative social interaction, and self-rated health. Evidence from two United States nationwide longitudinal surveys. Ageing and Society, 28(7), 1001-1023.

Kriwy, P., \& Nisic, N. (2012). Gesundheit als Ressource. In A. Knecht (Hrsg.), Ressourcen im Sozialstaat und in der Sozialen Arbeit. Ein Handbuch, (S. 89-101). Stuttgart: Kohlhammer.

Labianca, G. (2014). Negative Ties in Organizational Networks. In D. Brass, G. Labianca, A. Mehra, D. S. Halgin \& S. P. Borgatti (Hrsg.), Research in the Sociology of Organizations, (S. 239-259). Bradford, UK: Emerald.

Labianca, G., \& Brass, D. J. (2006). Exploring the social ledger. Negative relationships and negative asymmetry in social networks in organizations. Academy of Management Review, 31(3), 596-614.

Laireiter, A.-R., \& Lettner, K. (1993). Belastende Aspekte sozialer Netzwerke und sozialer Unterstützung. In A.-R. Laireiter (Hrsg.), Soziales Netzwerk und soziale Unterstützung. Konzepte, Methoden und Befunde (S. 101-114). Bern: Huber.

Lamnek, S., Luedtke, J., Ottermann, R., \& Vogl, S. (2013). Tatort Familie. Häusliche Gewalt im gesellschaftlichen Kontext. Wiesbaden: Springer VS.

Leary, M. R. (2005). Sociometer theory and the pursuit of relational value. Getting to the root of self-esteem. European Review of Social Psychology, 16(1), 75-111.

Leary, M. R. (2012). Sociometer theory. In A. W. Kruglanski, E. T. Higgins, \& P. A. M. van Lange (Hrsg.), Theories of social psychology (S. 141-159). Los Angeles: Sage. 
Lin, N. (2000). Social resources and social action. A theory of social capital. New York: Cambridge University Press.

Lincoln, K. D., Taylor, R. J., Bullard, K. M., Chatters, L. M., Woodward, A. T., Himle, J. A., \& Jackson, J. S. (2010). Emotional support, negative interaction and DSM IV lifetime disorders among older African Americans: Findings from the National Survey of American Life (NSAL). International Journal of Geriatric Psychiatry, 25(6), 612-621.

Lüscher, K. (2011). Über Ambivalenz. Forum der Psychoanalyse, 27(4), 323-327.

McCubbin, H. I., \& Patterson, J. M. (1983). The family stress process. The double ABCX model of adjustment and adoption. In H. I. McCubbin \& J. M. Patterson (Hrsg.), Social stress and the family (S. 7-37). New York: Haworth Press.

Nolting, H.-P. (1998). Konflikt. In S. Grubitzsch \& K. Weber (Hrsg.), Psychologische Grundbegriffe. Ein Handbuch (S. 552-554). Reinbek: Rowohlt.

Ntounas, P., Katsouli, A., Efstathiou, V., Pappas, D., Chatzimanolis, P., Touloumis, C., Papageorgiou, C., \& Douzenis, A. (2018). Comparative study of aggression - Dangerousness on patients with paranoid schizophrenia. Focus on demographic data, PANSS, drug use and aggressiveness. International Journal of Law and Psychiatry, 60, 1-11.

Offer, S. (2012). The burden of reciprocity. Processes of exclusion and withdrawal from personal networks among low-income families. Current Sociology, 60(6), 788-805.

Offer, S. \& Fischer, C. S. (2018). Difficult People. Who is percieved to be demanding in personal networks and why are they there? American Sociological Review, 83(1), 111-142.

Orth-Gomér, K. (2007). Psychosocial and behavioral aspects of cardiovascular disease prevention in men and women. Current Opinion in Psychiatry, 20(2), 147-151.

Orth-Gomér, K. (2009). Are social relations less health protective in women than in men? Social relations, gender, and cardiovascular health. Journal of Social and Personal Relationships, 26(1), 63-71.

Pape, J. C., \& Binder, E. B. (2014). Psychotrauma als Risiko für spätere psychische Störungen. Epigenetische Mechanismen. Der Nervenarzt, 85(11), 1382-1389.

Perry, B. L., Pescosolido, B. A., \& Borgatti, S. P. (2018). Egocentric network analysis. Foundations, methods, and models (Structural analysis in the social sciences, Bd. 44). Cambridge: Cambridge University Press.

Peuckert, R. (2006). Abweichendes Verhalten und soziale Kontrolle. In H. Korte \& B. Schäfers (Hrsg.), Einführung in Hauptbegriffe der Soziologie (S. 105-125). Wiesbaden: VS Verlag.

Phan, M. B., Blumer, N., \& Demaiter, E. I. (2009). Helping hands. Neighborhood diversity, deprivation, and reciprocity of support in non-kin networks. Journal of Social and Personal Relationships, 26(6-7), 899-918.

Piff, P. K., Kraus, M. W., Côté, S., Cheng, B. H., \& Keltner, D. (2010). Having less, giving more. The influence of social class on prosocial behavior. Journal of Personality and Social Psychology, 99(5), 771-784.

Puhl, R. M., \& Brownell, K. D. (2006). Confronting and coping with weight stigma. An investigation of overweight and obese adults. Obesity, 14(10), 1802-1815.

Rensing, L. (2013). Mensch im Stress. Psyche, Körper, Moleküle. Berlin: Springer VS.

Rook, K. S. (1984). The negative side of social interaction. Impact on psychological wellbeing. Journal of Personality and Social Psychology, 46(5), 1097-1108. 
Rook, K. S. (1998). Investigating the positive and negative sides of personal relationships. Through a glass darkly? In W. R. Cupach \& B. H. Spitzberg (Hrsg.), The dark side of close relationships (S. 369-393). New York: Routledge.

Rook, K. S. (2015). Social Networks in later life. Weighing positive and negative effects on health and well-being. Current Directions in Psychological Science, 24(1), 45-51.

Rook, K. S., August, K. J., Stephens, M. A. P., \& Franks, M. M. (2011). When does spousal social control provoke negative reactions in the context of chronic illness? The pivotal role of patients' expectations. Journal of Social and Personal Relationships, 28(6), 772-789.

Rusbult, C. E., \& Zembrodt, I. M. (1983). Responses to dissatisfaction in romantic involvements. A multidimensional scaling analysis. Journal of Experimental Social Psychology, 19(3), 274-293.

Rüssmann, K., Kopp, J., \& Hill, P. B. (2015). Macht, Arbeitsteilung, Konflikt, Konfliktstile und Gewalt in Partnerschaften. In P. B. Hill \& J. Kopp (Hrsg.), Handbuch Familiensoziologie (S. 487-527). Wiesbaden: Springer VS.

Seeman, T. E., Gruenewald, T. L., Cohen, S., Williams, D. R., \& Matthews, K. A. (2014). Social relationships and their biological correlates. Coronary Artery Risk Development in Young Adults (CARDIA) study. Psychoneuroendocrinology, 43, 126-138.

Seidel, E.-M., Kogler, L., Moser, E., Windischberger, C., Kryspin-Exner, I., Gur, R. C., Habel, U., \& Derntl, B. (2013). Weiblicher vs. männlicher Stresstyp? Ein aktueller Überblick zur neuropsychologischen Stressforschung. Zeitschrift für Psychiatrie, Psychologie und Psychotherapie, 61(2), 71-79.

Siegrist, J. (2018). Soziale Stressoren und stressbedingte Erkrankungen. In R. Fuchs \& M. Gerber (Hrsg.), Handbuch Stressregulation und Sport (S. 79-92). Berlin: Springer.

Simmel, G. (1908). Soziologie. Untersuchungen über die Formen der Vergesellschaftung. Kapitel IV: Der Streit. http://socio.ch/sim/soziologie/index.htm. Zugegriffen: 28. Febr. 2019.

Sneed, R. S., \& Cohen, S. (2014). Negative social interactions and incident hypertension among older adults. Health Psychology, 33(6), 554-565.

Stafford M., McMunn, A., Zaninotto, P., \& Nazroo, J. (2011). Positive and negative exchanges in social relationships as predictors of depression. Evidence from the English Longitudinal Study of Aging. Journal of Aging and Health, 23(4), 607-628.

Sykes, H., \& McPhail, D. (2008). Unbearable lessons. Contesting fat phobia in physical education. Sociology of Sport Journal - Special Issue on Social Construction of Fat, 25(1), 66-96.

Tanne, D., Goldbourt, U., \& Medalie, J. H. (2004). Perceived family difficulties and prediction of 23-year stroke mortality among middle-aged men. Cerebrovascular Diseases, 18(4), 277-282.

Taylor, S. E., Klein, L. C., Lewis, B. P., Gruenewald, T. L., Gurung, R. A., \& Updegraff, J. A. (2000). Biobehavioral responses to stress in females. Tend-and-befriend, not fightor-flight. Psychological Review, 107(3), 411-429.

Tippett, N., \& Wolke, D. (2014). Socioeconomic status and bullying. A meta-analysis. American Journal of Public Health, 104(6), e48-e59.

Tsuno, K., Kawakami, N., Tsutsumi, A., Shimazu, A., Inoue, A., Odagiri, Y., Yoshikawa, T., Haratani, T., Shimomitsu, T., \& Kawachi, I. (2015). Socioeconomic determinants of bullying in the workplace. A national representative sample in Japan. PloS one, 10(3), $\mathrm{e} 0119435$. 
Uchino, B. N. (2009). Understanding the Links Between Social Support and Physical Health. A Life-Span Perspective With Emphasis on the Separability of Perceived and Received Support. In Perspectives on Psychological Science, 4(3), 236-255.

von Dawans, B., \& Heinrichs, M. (2018). Physiologische Stressreaktion. In R. Fuchs \& M. Gerber (Hrsg.), Handbuch Stressregulation und Sport (S. 67-78). Berlin: Springer.

Wright, B. L., \& Loving, T. J. (2011). Health implications of conflict in close relationships. Social and Personality Psychology Compass, 5(8), 552-562.

Open Access Dieses Kapitel wird unter der Creative Commons Namensnennung 4.0 International Lizenz (http://creativecommons.org/licenses/by/4.0/deed.de) veröffentlicht, welche die Nutzung, Vervielfältigung, Bearbeitung, Verbreitung und Wiedergabe in jeglichem Medium und Format erlaubt, sofern Sie den/die ursprünglichen Autor(en) und die Quelle ordnungsgemäß nennen, einen Link zur Creative Commons Lizenz beifügen und angeben, ob Änderungen vorgenommen wurden.

Die in diesem Kapitel enthaltenen Bilder und sonstiges Drittmaterial unterliegen ebenfalls der genannten Creative Commons Lizenz, sofern sich aus der Abbildungslegende nichts anderes ergibt. Sofern das betreffende Material nicht unter der genannten Creative Commons Lizenz steht und die betreffende Handlung nicht nach gesetzlichen Vorschriften erlaubt ist, ist für die oben aufgeführten Weiterverwendungen des Materials die Einwilligung des jeweiligen Rechteinhabers einzuholen.



